\title{
Evolution of second trimester low implanted placenta to previa at term: a prospective cohort study
}

\author{
Omar M. Shaaban', Hamdy A. Ahmed², Mohammed K. Ali' ${ }^{1}$, Ahmed M. Abbas ${ }^{1 *}$, \\ Ahmed Nasr ${ }^{1}$, Ahmed M. Makhlouf ${ }^{1}$
}

\begin{abstract}
${ }^{1}$ Department of Obstetrics and Gynecology, Faculty of Medicine, Assiut University, Assiut, Egypt
\end{abstract}
${ }^{2}$ Department of Obstetrics and Gynecology, Sohag General Hospital, Sohag, Egypt

Received: 21 August 2018

Accepted: 27 September 2018

\section{*Correspondence:}

Dr. Ahmed M. Abbas,

E-mail: bmr90@hotmail.com

Copyright: (c) the author(s), publisher and licensee Medip Academy. This is an open-access article distributed under the terms of the Creative Commons Attribution Non-Commercial License, which permits unrestricted non-commercial use, distribution, and reproduction in any medium, provided the original work is properly cited.

\begin{abstract}
Background: The objective of this study is to identify the percentage of low implanted placenta (LIP) at second trimester of pregnancy and identify the risk factors of its persistence to placenta previa (PP) at term.

Methods: Through a registered prospective cohort study conducted at tertiary hospital in Upper Egypt, authors screened all pregnant ladies comes to present facility for antenatal second trimester USG between 18-24 weeks gestation. All participants interviewed for detailed risk factors of placenta previa. Those diagnosed to have a LIP $(\leq 1.5 \mathrm{~cm}$ from the internal os) had had TVS to confirm the exact distance between the lower edge of the placenta and the internal os. Serial USG had been done every 4 weeks up to delivery to measure the same distance. The primary outcome was the percentage of LIP at 18-24 weeks. Logistic regression analysis was performed to predict the risk factors for PP at term.

Results: Through screening of 1000 pregnant lady, LIP had been identified in (52 cases) 5.2\% of pregnant women between 18-24 weeks. This percentage dropped gradually to reach $1.3 \%$ at 36 weeks of gestation and at time of delivery. The logistic regression analysis demonstrated that the distance between the internal os and the lower edge of the placenta between 18-24 weeks was the single significant variable associated with PP at term ( $<<0.001$, odds ratio $0.319,95 \%$ CI 0.20-0.50). However, excluding the distance from the regression model demonstrated other risk factors as previous miscarriage, previous cesarean section (CS), and history of multiple pregnancies and history of previous PP.

Conclusions: About 5.0\% of pregnant women have LIP at the second trimester of pregnancy (18-24 weeks) and only $25.0 \%$ of them remain placenta previa at term. A cut-off value of $10 \mathrm{~mm}$ between the internal os and the lower edge of the placenta is the most important predictor of development of PP.
\end{abstract}

Keywords: Low implanted placenta, Placenta previa, Ultrasound

\section{INTRODUCTION}

Placenta previa (PP) is a placenta inserted into the lower uterine segment; it is responsible for $0.03 \%$ of maternal mortality and $8.1 \%$ of perinatal mortality in the developed world and much more in developing countries. ${ }^{1,2}$ Placenta previa is a major cause of maternal hemorrhage, a complication that is directly correlated the number of previous CS done for the same lady. ${ }^{3}$

The rate of CS is increased and increasing worldwide, reaching about $52 \%$ in Egypt in the last demographic. ${ }^{4}$ Although various studies have tried to address risk factors of PP; the exact etiology of PP still remains obscure. ${ }^{5}$ 
Several risk actors have been identified as strong contributors to PP development like high parity, advanced maternal age, history of previous CS and history of previous PP. ${ }^{6}$ Unlike first-trimester bleeding, the PP bleeding is usually due to abnormal placental implantation which usually started in the third trimester. ${ }^{7}$

A high number of women with asymptomatic PP or LIP could be detected by ultrasonography when performed at the mid trimester. ${ }^{8}$ However; a high proportion of them, about $66 \%$ will be migrated especially in marginal, incomplete or low laying PP. ${ }^{9}$ So the guidelines recommend follow-up at 32 weeks' gestation to assess for persistence of placenta previa. $^{10}$ Transvaginal ultrasonography has an important role in predicting PP at term by determining the distant between the internal cervical os and the lower placental edge at early second trimester. ${ }^{11}$ However; there is no consensus about the exact distance, which can give the obstetricians a high sensitivity rate of diagnosis. ${ }^{12}$ Additionally, the maternal characteristics may be also having a role in prediction of PP.

The current study aims to identify the percentage of low implanted placenta (LIP) at second trimester of pregnancy and identify the risk factors of its persistence to $\mathrm{PP}$ at term.

\section{METHODS}

The current study was a clinically registered prospective cohort study (NCT03321435) compassing the percentage of LIP at 18-24 weeks and the risk factors contributed to progression to PP at term. LIP between 18-24 weeks is defined as the overlap of a placental edge on the lower uterine segment ( $15 \mathrm{~mm}$ or less from the internal os). ${ }^{13}$

The Institutional review Board of Assiut Faculty of Medicine hade approved the study. The study participants were recruited from the outpatient clinic of Woman's Health Hospital, Assiut University, Egypt from $1^{\text {st }}$ of October 2016 to $30^{\text {th }}$ of March 2017. Women who met the eligibility criteria of the study were invited to participate and only those who signed the informed consent were recruited. The consent form included using their medical data anonymously for research purpose.

\section{Inclusion criteria}

Authors included in present study women aged between 20-35 years, with BMI $18-22 \mathrm{~kg} / \mathrm{m}^{2}$ who had a singleton baby between 18-24weeks gestation. Gestational age established by either reliable dates or confirmed by early ultrasound.

\section{Exclusion criteria}

However; authors excluded women refused to perform trans-vaginal ultrasound (TVS) in the second trimester and who were living away from present hospital so the follow up would be quite difficult.

Each patient was subjected to interview questioner of detailed personal and obstetric history, including: age, parity, number of normal deliveries, number abnormal deliveries, number of multiple pregnancies, number of pervious abortions, smoking, previous uterine operations and previous PP. All pregnant ladies scanned by trans abdominal ultrasound (TA-USG) using a 3- to 5-MHz abdominal probe during this visit to identify cases suspicious of LIP.

Authors cared about bladder over distension during TAS to avoid false positive results. Those who found to have a placental tissue within $15 \mathrm{~mm}$ from the internal os by TA-USG were underwent TVS-USG (Medison BW 128, Korea) using a 4- to 7-MHz endo-vaginal probe for accurate measuring of the distance between the lower edge of the placenta and the internal. Additionally, the placental location either anterior or posterior was recorded. All ultrasound examinations were done by a single sonographer to decrease the interobserver errors. Pregnant women with LIP women were encouraged to follow up every 4 weeks at present facility till time of delivery.

During each follow-up visit TVS scan was done and the distance between the lower edge of the placenta and the internal os was reported. Definition of LIP was changed to become $50 \mathrm{~mm}$ from the internal os starting from 28 weeks gestation. ${ }^{14}$ Other sonographic findings had been assessed by TAS. All data were collected in a specially designed data collection sheet that included patients contact details, address and cell phone to facilitate their follow-up.

The primary outcome of the study was the percentage of LIP at mid trimester (18-24 weeks). Secondary outcomes included the percentage of progression of LIP at mid trimester to PP at term and the potential risk factors for prediction of development of PP.

PP at term means placenta within $50 \mathrm{~mm}$ from the internal os. ${ }^{14}$ Additionally, the placental condition at the time of delivery and the degree of its adherence to the lower uterine segment as well as any maternal complications at the time of delivery had also been recorded.

Sample size was calculated based on the primary outcome (the percentage of LIP at mid trimester). A previous study reported that the percentage of LIP at mid trimester was about $4.0 \% .{ }^{15}$ Hypothesizing the population size is 100,0000 , percentage of frequency of outcome factor in present population is $4.0 \%$ and confidence limits is $5.0 \%$, a total sample size of at least 55 patients will be needed assuming a rate of loss to follow-up of $10 \%$ (Epiinfo TM, CDC, USA). 


\section{RESULTS}

One thousand five hundred pregnant women between 18 24 weeks gestation were initially interviewed for inclusion in the study. Five hundred women were excluded; 320 because of refusing to perform TVS during pregnancy and 180 because they were livening away from present facility which make the subsequent followup up to delivery not feasible.

Between 18-24 weeks gestation, authors found 52 women (5.2\%) had had LIP (within $15 \mathrm{~mm}$ from the internal os, while the majority of cases $(94.8 \%)$ had a higher placenta. ${ }^{13}$ By 28 weeks; authors found that only 22 women out of 52 women still had their placentae classified as LIP, taking in consideration that the definition of LIP changes from the start of the $3^{\text {rd }}$ trimester to $50 \mathrm{~mm} .{ }^{14}$ Furthermore, by 32 weeks; only 14 out of 22 women still had their placentae low implanted, those decreased to 13 women at 36 weeks and at delivery (Figure 1, the study flow chart). Two cases (3.8\%) had been diagnosed preoperatively to be suspicious of abnormal invasion (placenta accreta).

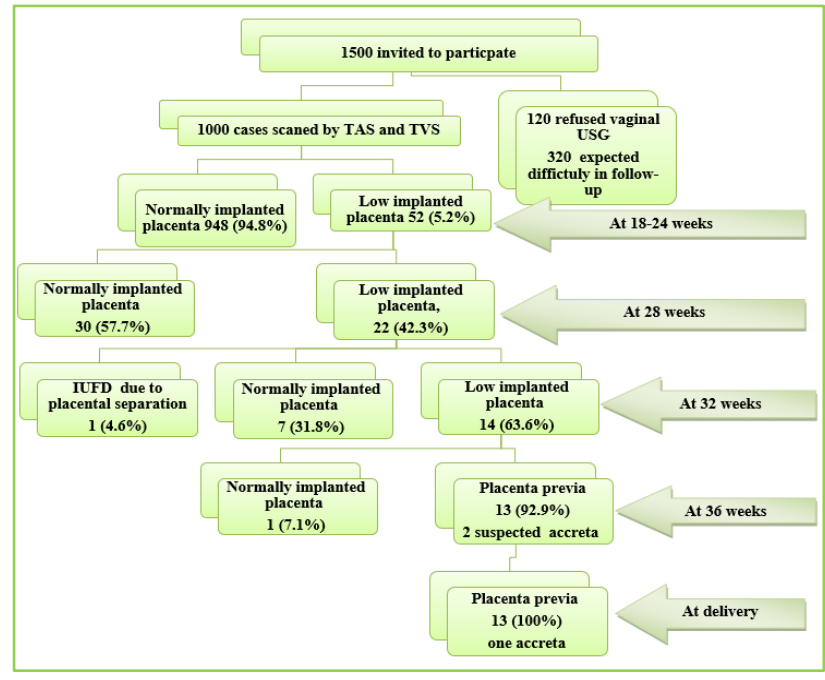

Low implanted placenta between 18-24 weeks means placenta within $15 \mathrm{~mm}$ from the internal os (SOGC, 2007). Low implanted placenta at $3^{\text {rd }}$ trimester means placenta within 50 $\mathrm{mm}$ from the internal os (McDonald et al, 1997).

Figure 1: Study flow chart.

Table 1: The socio-demographic characteristics between LIP women and HP women between 18-24 weeks gestation.

\begin{tabular}{|c|c|c|c|}
\hline Clinical characteristics & LIP $(n=52)$ & HP $(n=948)$ & P value \\
\hline Age, mean \pm SD & $26.96 \pm 4.93$ & $26.87 \pm 4.77$ & 0.888 \\
\hline \multicolumn{4}{|l|}{ Residency, n (\%) } \\
\hline Sohag & $30(57.7 \%)$ & $522(55.1 \%)$ & \multirow{2}{*}{0.710} \\
\hline Assiut & $22(42.3 \%)$ & $426(44.9 \%)$ & \\
\hline Number of previous CS, n (\%) & $13(25 \%)$ & $131(13.8 \%)$ & $0.025^{*}$ \\
\hline Number of previous multiple pregnancy, $\mathrm{n}(\%)$ & $8(15.4 \%)$ & $35(3.7 \%)$ & $0.001 *$ \\
\hline History of previous placenta previa, n (\%) & $1(1.9 \%)$ & $2(0.2 \%)$ & 0.148 \\
\hline Parity, mean \pm SD & $1.94 \pm 1.35$ & $1.97 \pm 1.68$ & 0.895 \\
\hline Number of previous miscarriages, $\mathrm{n}(\%)$ & $0.48 \pm 0.80$ & $0.56 \pm 0.81$ & 0.497 \\
\hline \multicolumn{4}{|l|}{ Placental position, $\mathbf{n}(\%)$} \\
\hline Anterior & $30(57.5 \%)$ & $638(67.3 \%)$ & 0.152 \\
\hline Posterior & $22(42.5 \%)$ & $310(32.7 \%)$ & \\
\hline Distance of the internal OS $(\mathrm{mm})$, mean \pm SD & $6.90 \pm 5.65$ & $27.25 \pm 7.48$ & $0.001 *$ \\
\hline
\end{tabular}

Table 1 shows that there were no significant differences between women with LIP and those with a higher placenta at 18-24 weeks in socio-demographic data except in history of previous CS which was much higher in LIP women than women with a higher placenta $(25 \%$ versus $13.8 \%$; respectively, $\mathrm{p}=0.025$ ). As regard history of multiple pregnancy; the figures are $15 \%$ in LIP women versus $4 \%$ in a high placenta women; respectively with statistically significant difference $(\mathrm{p}=0.001)$ (Table 1).
Seventy five percent of LIP between 18-24 weeks became a high implanted placenta at time of delivery. In the other hand; $25 \%$ of cases persist as PP at time of delivery. The possible risk factors for progression of LIP between 18-24 weeks to PP at term were calculated by multivariate logistic regression analysis. Authors found that the only significant predictive factor was the distance between the internal os and the lower edge of the placenta (Table 2). 
Table 2: Multivariate logistic regression analysis for possible risk factors for LIP between 18-24 weeks including the distance from the internal os.

\begin{tabular}{|l|ll|}
\hline Parameter & Multivariate analysis \\
\hline OR (95\% CI) & P value \\
\hline Age & 1.058 & 0.385 \\
\hline Residency & 0.948 & 0.472 \\
\hline CS & 4.476 & 0.089 \\
\hline Multiple pregnancy & 0.843 & 0.636 \\
\hline Parity & 0.843 & 0.404 \\
\hline History of PP & 30.885 & 1.000 \\
\hline Miscarriage & 0.558 & 0.895 \\
\hline Distance from IO & 0.319 & $<0.001^{*}$ \\
\hline
\end{tabular}

*statistical significance if p-Value less than 0.05 , CS cesarean section, PP placenta previa, IO internal os.

Excluding the distance from the internal os from the regression model resulted in the appearance of the effect of other significant factors namely previous miscarriage, previous CS, history of multiple pregnancies and history of previous PP (Table 3 ).

Table 3: Multivariate logistic regression analysis for possible risk factors for LIP between 18-24 weeks excluding the distance from the internal os.

\begin{tabular}{|c|c|c|}
\hline Parameter & \multicolumn{2}{|c|}{ Multivariate analysis } \\
\hline & OR $(95 \%$ CI $)$ & P value \\
\hline Age & 1.019 & 0.531 \\
\hline Residency & 0.995 & 0.987 \\
\hline $\mathrm{CS}$ & 0.404 & $0.010 *$ \\
\hline Multiple pregnancy & 0.147 & $<0.001 *$ \\
\hline Parity & 0.918 & 0.371 \\
\hline History of PP & 0.065 & $0.050 *$ \\
\hline Miscarriage & 0.615 & $0.041 *$ \\
\hline
\end{tabular}

*statistical significance if p-value less than 0.05 , CS cesarean section, PP placenta previa.

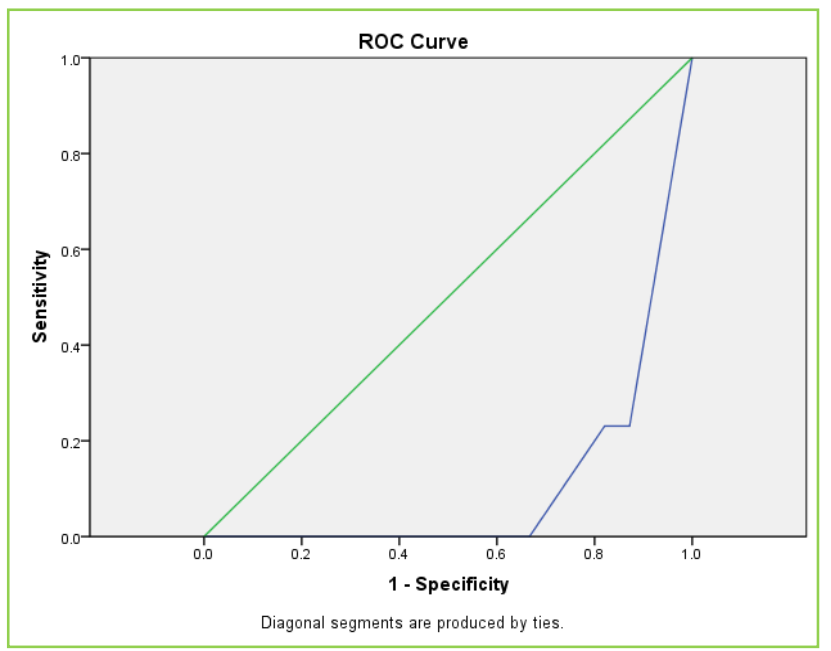

Figure 2: Receiver operator characteristics (ROC) curve analysis for predictive value of internal os distance at 18 weeks for the 52 cases with PP to complete as PP.
Finally; authors constructed receiver operating characteristic (ROC) curves to evaluate the sensitivity and specificity of the most significant predictor revealed by logistic regression which was (distance from IO). Authors found that the most suitable cut-off point of internal os distance at 18-24 weeks predicting the progression of LIP to PP at term is $10 \mathrm{~mm}$, with a sensitivity of $95 \%$ and a specificity of $83 \%$ (Figure 2).

Complications that encountered at the time of delivery in cases of PP were 18 cases $(34.6 \%)$ of cases had received antenatal or intra-natal blood transfusion, 14 (26.7\%) cases had developed post-partum hemorrhage and only one case proved to be abnormally adherent at the time of cesarean delivery.

\section{DISCUSSION}

Second trimester screening of a thousand of pregnant women between 18-24 weeks diagnosed LIP as defined by $15 \mathrm{~mm}$ or less from the internal os in $5.2 \%$ of cases. Prospective follow-up of these cases dropped with the rate nto $1.3 \%$ of cases to become overt PP at term and during delivery. This is confirming the fortunate upward change of placental location with advancement of gestational age placental migration. Authors studded the potential predictors of this favorable placental migration and identified that the distance between the internal os and the lower edge of the placenta as the most important predictor at the second trimester of pregnancy. Other contributors included previous history of miscarriage, previous CS, history of multiple pregnancies and history of previous PP had been also identified.

Placenta previa is a life threatening obstetric complication; however, its proper anticipation and preparation of pregnant women with PP is markedly decreases the maternal morbidity and mortality. ${ }^{16}$ Present detection rate of LIP $(5.2 \%)$ is quiet similar to what have been found by Rosati and colleagues (4.9\%)between 1016 weeks gestation. ${ }^{17}$ Additionally, Mustafa et al reported also an incidence of $3.9 \%$ between 20-24 weeks gestation. ${ }^{15}$ Again; Shukunami in his study reported an incidence of $6.0 \%$ at $12-15$ weeks of pregnancy. ${ }^{18}$ Variance in the gestational age of scanning and the type of included participants may be behind this minor difference. On the other hand, other studies found much lower incidence ranged from 0.66 to 1.1 between $15-20$ weeks. ${ }^{19,20}$ Difference in the span of time at which different studies had been done may be the cause of lower detection rate in some studies as CS rate is different. Moreover, present rate of detection of LIP was $2.2 \%$ at 28 weeks scan. This is in accordance to Obata and colleagues of $2.8 \%$ who scanned patients at 29 weeks gestation. $^{21}$ With advancement of pregnancy, by 36 weeks of gestation the rate of PP in present cohort was $1.3 \%$ and the same rate observed at the time of CS at term. This is near what has been recently reported by Omakanye who reported $1.6 \%$ incidence of PP among 10,250 deliveries. $^{22}$ On the contrary; much lower 
incidence was reported by Kollmann $(0.36 \%$ in 2003 and $0.74 \%$ in 2011 ) which could be explained by the parallel increase in CS deliveries from 24.2 to $31.9 \% .^{23}$

These findings may point to the presence of an element of change in the placental location with the progress of pregnancy. The placental trophotropism which means degeneration of thin placental margins and rapid growth of the lower uterine segment may explain this phenomenon. ${ }^{24}$ From the above studies, it can be settled that the rate of detection of LIP decrease with the advancement of gestational age and there is no consensus about the cut of value of distance early in pregnancy that can predict the LIP at term. Moreover, it seems that the reported incidence of PP is increasing year after year and this is parallel to the increase in the CS rate.

The distance from the internal os measured between 18 24 weeks was the only significant risk predictor. This was in accordance to Kacey and colleagues who found that the likelihood of resolution of LIP detected after 14 weeks gestation was affected by only gestational age and distance from the internal os at time of diagnosis. ${ }^{25}$

Present study demonstrated that the most significant cutoff points for predicting the persistent of second trimester LIP to PP was $10 \mathrm{~mm}$. This cut of value had a very high sensitivity $(95 \%)$ and relatively high specificity (83\%). Authors are in agreement with Taipale and colleagues who agreed that persistence of PP is extremely unlikely if the distance is more than $10 \mathrm{~mm} .^{26}$ On the other hand, Mustafa and colleagues reported a distance that a distance of $2.4 \mathrm{~cm}$ between 11-14 week, gave $80.0 \%$ probability of $\mathrm{PP}$ at term. ${ }^{15}$ The difference in the cut value may be secondary to the earlier scan of LIP in Mustafa and colleagues study in addition to the difference in the other risk factors.

Present study had its limitation, as authors followed up only women who diagnosed to have LIP based on present criteria up to delivery not all the scanned women. Based on present study authors do recommend second trimester scan of all pregnant women for exclusion of LIP. Close follow up of those with placentae $10 \mathrm{~cm}$ or less from the internal os as about one quarter of them will develop PP. Further studies are needed for early detection of second trimester LIP with the cut of value of $10 \mathrm{~mm}$ is greatly recommended.

\section{CONCLUSION}

About $5.0 \%$ of pregnant women have LIP at the second trimester of pregnancy (18-24 weeks) and only $25.0 \%$ of them remain placenta previa at term. A cut-off value of $10 \mathrm{~mm}$ between the internal os and the lower edge of the placenta is the most important predictor of development of PP.

Funding: No funding sources Conflict of interest: None declared
Ethical approval: The study was approved by the Institutional Ethics Committee

\section{REFERENCES}

1. Royal College of Obstetricians and Gynaecologists. Placenta praevia, placenta praevia accreta and vasa praevia: diagnosis and management. Green-top Guideline No. 27. London: RCOG; 2011.

2. Umazume $\mathrm{T}$, Hayasaka $\mathrm{S}$, Kato $\mathrm{F}$, Ishikawa $\mathrm{S}$, Morikawa M, Minakami H. Sudden maternal hypoxemia during elective cesarean section in a woman with placenta previa. Clin Case Rep. 2017;5(10):1668-71.

3. Kollmann M, Gaulhofer J, Lang U, Klaritsch P. Placenta praevia: incidence, risk factors and outcome. J Matern Fetal Neonatal Med. 2016;29(9):1395-8.

4. Betrán AP, Ye J, Moller AB, Zhang J, Gülmezoglu AM, Torloni MR. The increasing trend in caesarean section rates: global, regional and national estimates: 1990-2014. PloS One. 2016;11(2):e0148343.

5. Royal College of Obstetricians and Gynaecologists. Placenta Praevia, Placenta Praevia Accreta and Vasa Praevia: Diagnosis and Management. Green-top Guideline No.27. London: RCOG; 2011.

6. Rosenberg T, Pariente G, Sergienko R, Wiznitzer A, Sheiner E. Critical analysis of risk factors and outcome of placenta previa. Arch Gynecol Obstet. 2011;284(1):47-51.

7. Sekiguchi A, Nakai A, Kawabata I, Hayashi M, Takeshita T. Type and location of placenta previa affect preterm delivery risk related to antepartum hemorrhage. Int J Med Sci. 2013;10(12):1683-8.

8. Bhide A, Thilaganathan B. Recent advances in the management of placenta previa. Curr Opin Obstet Gynecol. 2004;16(6):447-51.

9. Reddy UM, Abuhamad AZ, Levine D, Saade GR. Fetal imaging: executive summary. Obstet Gynecol. 2014;123:1070-82.

10. Eichelberger KY, Haeri S, Kessler DC, Swartz A, Herring A, Wolfe HM. Placenta previa in the second trimester: sonographic and clinical factors associated with its resolution. Am J Perinatol. 2011;28:735-40.

11. Lauria MR, Smith RS, Treadwell MC, Comstock $\mathrm{CH}$, Kirk JS, Lee W, et al. The use of secondtrimester transvaginal sonography to predict placenta previa. Ultrasound Obstet Gynecol. 1996;8(5):33740.

12. Durst JK, Tuuli MG, Temming LA, Hamilton O, Dicke JM. Resolution of a low-lying placenta and placenta previa diagnosed at the mid trimester anatomy scan. J Ultrasound Med. 2018.

13. SOGC clinical practice guideline. Diagnosis and Management of Placenta Previa. No. 189, March 2007.

14. Macdonald AA, Fowden AL. Microscopic anatomy of the ungulate placenta. Equine Vet J Suppl. 1997;24:7-13. 
15. Mustafa SA, Brizot ML, Carvalho MH, Watanabe L, Kahhale S, Zugaib M. Transvaginal ultrasonography in predicting Placenta previa at delivery: a longitudinal study. Ultrasound Obstet Gynecol. 2002;20:356-9.

16. Daskalakis G, Simou M, Zacharakis D, Detorakis S, Akrivos N, Papantoniou N, et al. Impact of placenta previa on obstetric outcome. Int J Gynaecol Obstet. 2011;114:238-41.

17. Rosati P, Guariglia L. Clinical significance of placenta previa detected at early routine transvaginal scan. J Ultrasound Med. 2000;19(8):581-5.

18. Shukunami K, Nishijima K, Kurodawa T, Tajima K, Kamitani N, Yoshida Y, et al. A small-angled thin edge of the placenta predicts abnormal placenation at delivery. J Ultrasound Med. 2005;24:331-5.

19. Becker RH, Vonk R, Mende BC. The relevance of placental location at 20-23 gestational weeks for prediction of Placenta previa at delivery: evaluation of 8650 cases. Ultrasound Obstet Gynecol. 2001;17:496-501.

20. Bhide A, Prefumo F, Moore J, Hollis B, Thilaganathan B. Placental edge to internal os distance in the late third trimester and mode of delivery in placenta praevia. BJOG. 2003;110(9):860-4.

21. Obata S, Ishiharak. Ultrasonographic study and lowlying placenta and it's clinical significance. Nippon Sanka Fujinka Gakkai Zasshi. 1993;45:1115-8.
22. Omokanye LO, Olatinwo AW, Salaudeen AG, Ajiboye AD, Durowade KA. A 5-year review of pattern of placenta previa in Ilorin, Nigeria. Int $\mathbf{J}$ Health Sci. 2017;11(2):35

23. Kollmann M, Gaulhofer J, Lang U, Klaritsch P. Placenta praevia: incidence, risk factors and outcome. Ultrasound Med-Eur J Ultrasound. 2013;34(S 01):WS_SL6_01.

24. Fidan U, Ulubay M, Bodur S, FerdiKinci M, EmreKaraşahiN K, Cemal Yenen M. The effect of anatomical placental location on the third stage of labor. Clin Anat. 2017;30(4):508-11.

25. Kacey E, Honor M, Anthony S, Dave K, Amy H. Second trimester placenta previa and resolution patterns: a four year institutional review. JOG. 2009;201(6):135.

26. Taipale P, Hiilesmaa V, Ylostalo P. Diagnosis of Placenta previa by transvaginal sonographic screening at $12-16$ weeks in a non-selected population. Obstet Gynecol. 1997;89:364-7.

Cite this article as: Shaaban OM, Ahmed HA, Ali MK, Abbas AM, Nasr A, Makhlouf AM. Evolution of second trimester low implanted placenta to previa at term: a prospective cohort study. Int J Reprod Contracept Obstet Gynecol 2018;7:4330-5. 